\title{
Placental Growth Factor Mediates Crosstalk Between Lung Cancer Cells and Tumor- Associated Macrophages in Controlling Cancer Vascularization and Growth
}

\author{
Changjun $\mathrm{He}^{\mathrm{a}} \quad$ Kaibin Zhu Xue Bai $^{\mathrm{a}} \quad$ Yingbin Lib ${ }^{\mathrm{b}} \quad$ Dawei Sun ${ }^{\mathrm{a}} \quad$ Yaoguo Lang ${ }^{\mathrm{a}}$ \\ Jinfeng Ning ${ }^{\mathrm{a}}$ Fenglin Sun ${ }^{\mathrm{a}}$ Changfa $\mathrm{Qu}^{\mathrm{a}}$ Shidong $\mathrm{Xu}^{\mathrm{a}}$ \\ aDepartment of Thoracic Surgery, Harbin Medical University Cancer Hospital, Harbin, b Department of \\ Surgery, the First Affiliated Hospital of Harbin Medical University, Harbin, China
}

\section{Key Words}

Placental growth factor (PLGF) - Non-small cell lung cancer (NSCLC) - Tumor-associated macrophages (TAM) • Transforming growth factor $\beta 1$ (TGF $\beta 1$ )

\begin{abstract}
:
Background/Aims: Assistance with tumor-associated vascularization is needed for the growth and invasion of non-small cell lung cancer (NSCLC). Recently, it was shown that placental growth factor (PLGF) expressed by NSCLC cells had a critical role in promoting the metastasis of NSCLC cells. However, the underlying molecular mechanisms remain elusive. Methods: Here, we first established a NSCLC model in mice that allows us not only to isolate tumor cells from non-tumor cells in the tumor, but also to trace tumor cells in living animals. Levels of PLGF, its unique receptor Flt- 1 , as well as transforming growth factor $\beta 1$ (TGF $\beta 1$ ) was examined in tumor cells and tumor-associated macrophages (TAM) by RT-qPCR. A transwell well coculture system and HUVEC assay were applied to study the crosstalk between NSCLC cells and TAM. Results: NSCLC cells produced and secreted PLGF to signal to tumor-associated macrophages (TAM) through surface expression of Flt-1 on macrophages. In a transwell coculture system, PLGF secreted by NSCLC cells triggered macrophage polarization to a TAM subtype that promote growth of NSCLC cells. Moreover, polarized TAM seemed to secrete TGF $\beta 1$ to enhance the growth of endothelial cells in a HUVEC assay. Conclusion: The crosstalk between TAM and NSCLC cells via PLGF/FIt- 1 and TGF $\beta$ receptor signaling may promote the growth and vascularization of NSCLC.

(C) 2018 The Author(s)

Published by S. Karger AG, Basel
\end{abstract}

\section{Introduction}

Non-small cell lung cancer (NSCLC) is a prevalent malignant disease [1, 2]. Effective prevention and treatment of NSCLC largely relies on a thorough understanding of the molecular mechanisms underlying the growth and invasion of NSCLCs, which requires the

Shidong Xu

KARGER
Department of Thoracic Surgery, Harbin Medical University Cancer Hospital, 150 Hapin Road, Harbin (China)

Tel. +8645185718180, E-Mail cmhzr37@yeah.net 
participation of cancer-related angiogenesis [3]. During the processes that control tumorassociated vascularization, cancer cells play an active role, in which they not only secrete proteinases to break through extracellular matrix [4], but also secrete angiogenic factors, resulting in augmentation of vessel formation inside and near the tumor [5].

Vascular endothelial growth factor (VEGF) family plays a critical role in the vasculogenesis and angiogenesis during both embryogenesis and adulthood [6]. So far, six secreted proteins: VEGF-a, VEGF-b, VEGF-c, VEGF-d, VEGF-e and placental growth factor (PLGF) have been found in the VEGF family [6]. Among these factors, PLGF was relatively newly detected, and recent evidence suggests that PLGF may be involved in the vascularization and angiogenesis under pathological circumstances [7-16]. VEGF receptor I (VEGFR1 or Flt-1) is the unique receptor for PLGF, and is mainly expressed on the surface of endothelial cells and monocyte/ macrophages [6].

Macrophages are traditionally regarded as white blood cells that engulf and digest body wastes, dead and dying cells as well as foreign substances [17-20]. However, in recent years, more and more functions of macrophages have been detected, leading to a classification of macrophages into 2 subtypes, including a classical phenotype termed "M1" macrophages and an alternatively polarized phenotype termed "M2" macrophages. M2 macrophages regulate humoral immunity and promote tissue repair [17-20]. Moreover, some macrophages inside the tumor are found to have characteristics of M2 but they enhances tumorigenesis, thus called tumor-associated macrophages (TAM) [21].

The M1 macrophages express high levels of nitric oxide synthase (iNOS) and reactive oxygen species (ROS), while M2 macrophages express CD163, CD206, arginase and CD301 [17-20]. A recent study showed that M2 macrophages were recruited to the injured pancreas, and produced and secreted high levels of transforming growth factor $\beta 1$ (TGF $\beta 1$ ) [22]. In another report, mesenchymal stem cells were transplanted to cure cardiac muscle injury, while the authors found that the MSCs not only recruit macrophages to enhance angiogenesis to promote regeneration of cardiac muscle, but also secrete BMP7 to contradict the fibrogenic effect of TGF $\beta 1$ by macrophages [23]. Interestingly, another several studies have demonstrated a role of PLGF in the crosstalk between tumor cells and macrophages $[15,24]$. These previous evidence encouraged us to investigate the role of TAM in NSCLC and the underlying molecular mechanisms. PLGF,

In the current study, we investigated the crosstalk between NSCLC cells and TAM through

\section{Materials and Methods}

\section{Protocol approval}

All the experimental methods in the current study have been approved by the research committee at Harbin Medical University. All the experiments have been carried out in accordance with the guidelines from the research committee at Harbin Medical University. All animal experiments were approved by the Institutional Animal Care and Use Committee at Harbin Medical University. Surgeries were performed in accordance with the Principles of Laboratory Care, supervised by a qualified veterinarian.

\section{NSCLC cell line and transduction}

A549 is a human NSCLC line that was purchased from American Type Culture Collection (ATCC, Rockville, MD, USA), and were cultured in Dulbecco's modified Eagle's media (DMEM, Invitrogen, Carlsbad, CA, USA) supplemented with 10\% fetal bovine serum (FBS, Invitrogen). A549 was first developed in 1972 by Dr. Giard through the removal and culturing of cancerous lung tissue in the explanted tumor a of 58-yearold caucasian male [25]. Inhibitor SB431542 (used at a dose of $10 \mu \mathrm{mol} / \mathrm{l}$ ) was purchased from Abcam (Cambridge, MA, USA), and used according to the introduction from the manufacturer. Recombinant PLGF was purchased from Sigma-Aldrich (St. Louis, MO, USA). Soluble Flt-1 (sFlt-1) was purchased from R\&D Biosystem (Los Angeles, CA, USA). A pAAV-CMV-LUC-2A-RFP vector (Clontech, Mountain View, CA, USA) was used to generate AAV, with a packaging plasmid carrying the serotype 6 rep and cap genes and a

\section{KARGER}




\section{Cellular Physiology Cell Physiol Biochem 2018;47:2534-2543 \begin{tabular}{l|l|l} 
and Biochemistry $10.1159 / 000491650$ & $\begin{array}{l}\text { (c) 2018 The Author(s). Published by S. Karger AG, Basel } \\
\text { www.karger.com/cpb }\end{array}$
\end{tabular}}

He et al.: PLGF Regulates Crosstalk Between NSCLC and TAM

helper plasmid carrying the adenovirus helper functions (Applied Viromics, LLC. Fremont, CA, USA). The AAVs were purified using $\mathrm{CsCl}$ density centrifugation and then titration was determined by a quantitative densitometric dot-blot assay. For cell transduction in vitro, the cells were incubated with AAV at a MOI of 100 for 12 hours. Stable transduced cells were selected by flow cytometry based on red fluorescent protein (RFP). Transduced cells were monitored in vivo by their expression of luciferase.

\section{NSCLC model in mice and tumor monitoring by bioluminescence}

Ten week-old male NOD/SCID mice (SLAC Laboratory Animal Co. Ltd, Shanghai, China) were used for transplantation of $10^{7} \mathrm{AAV}$-transduced/labeled A549 cells by tail vein injection. Bioluminescence was monitored 8 weeks after transplantation, using IVIS imaging system (Xenogen Corp., Alameda, CA, USA). Images were taken 10 minutes after intraperitoneal injection of $150 \mathrm{mg} / \mathrm{kg}$ luciferin (Sigma-Aldrich) and image analysis and bioluminescent quantification was performed using living image software (Xenogen Corp.).

Tumor digestion and cell sorting by flow cytometry

Implanted NSCLC in mice was resected, minced into small pieces, and then digested in the digestion media containing $40 \mathrm{mg} / \mathrm{dl}$ collagenase (Sigma-aldrich) and $0.05 \%$ trypsin (Sigma-Aldrich) at $37^{\circ} \mathrm{C}$ for 30 min. After the digestion, the cells were passed a $40 \mu \mathrm{m}$ filter for flow cytometric analysis, after incubation with conjugated CD31, F4/80 and CD163 antibodies (Becton-Dickinson Biosciences, San Jose, CA, USA). RFP was detected by direct fluorescence. Data were analyzed using FlowJo software (Flowjo LLC, Ashland, OR, USA).

\section{Isolation of bone marrow-derived macrophages}

Bones from 12-week-old male C57BL/6 mice were flushed with macrophage culture media (high-glucose DMEM supplemented with 10\% fetal bovine serum, $10 \%$ L929 conditioned media, 2\% MEM nonessential amino acids, 1\% l-glutamine, 1\% HEPES, 1\% penicillin-streptomycin and 0.1\% 2-mercaptoethanol, using a 26-gauge needle into a $50 \mathrm{ml}$ conical tube. The collected bone marrow was centrifuged at 1200 RPM for 5 minutes at $4^{\circ} \mathrm{C}$. The pellets were re-suspended and seeded at $10^{6}$ per well in 24 -well plates and cultured in a $37^{\circ} \mathrm{C}$ humid incubator. The cells were fed with fresh media every other day for 7 days, after which the cells were replenished with L929-free media for co-culture study.

\section{Transwell co-culture system}

Isolated bone-marrow-derived macrophages $\left(10^{5}\right)$ were co-cultured either with equal number of A549 cells $\left(10^{5}\right)$ with/without of $10 \mu \mathrm{mol} / \mathrm{l}$ SB431542, or with/without recombinant PLGF (100ng), or with/ without of $10 \mu \mathrm{g} / \mathrm{l} \mathrm{sFlt}-1$ in a transwell co-culture system with $0.4 \mu \mathrm{m}$ pore size of the transwell membrane. Two days after co-culture, the changes in A549 cell number were determined with an MTT assay, and the macrophage subtypes were determined by flow cytometry. The conditioned media of macrophages were used for HUVEC assay.

\section{MTT assay}

Cell growth was evaluated with a Cell Viability Kit (MTT, Roche, Indianapolis, IN, USA), according to the instruction of the manufacturer.

\section{$R T-q P C R$}

RT-qPCR was performed routinely. All primers ( $\beta$-actin, iNOS, PLGF and TGF $\beta 1$ ) were purchased from Qiagen (Hilden, Germany). Values of genes were normalized against $\beta$-actin and then compared with control.

\section{Collagen gel assay and quantification of vessel formation}

HUVECs were obtained from American Type Culture Collection (ATCC, Rockville, MD, USA), and cultured in Dulbecco's Modified Eagle Medium/F12 (DMEM/F12; Invitrogen) supplemented with 10mmol/l L-glutamine, 100U/ml penicillin, $100 \mu \mathrm{g} / \mathrm{ml}$ streptomycin, 10\% FBS and endothelial cell growth supplement (ECGS, Becton-Dickinson Biosciences) at $37^{\circ} \mathrm{C}$ and $5 \% \mathrm{CO}_{2}$. For collagen gel assay, the six-well tissue culture plate was coated with $1.5 \mathrm{mg} / \mathrm{ml}$ of rat tail collagen type 1 and $3 \mu \mathrm{g} / \mathrm{ml}$ of fibronectin (Becton-Dickinson Biosciences), after which $10^{5}$ HUVECs were seeded in each collagen-coated well and allowed to attach for 


\section{Cellular Physiology Cell Physiol Biochem 2018;47:2534-2543 \begin{tabular}{l|l} 
and Biochemistry & DOI: 10.1159/000491650 \\
Published o 2018 The Author(s). Published by S. Karger AG, Basel \\
www.karger.com/cpb
\end{tabular} \\ He et al.: PLGF Regulates Crosstalk Between NSCLC and TAM}

$1 \mathrm{~h}$. A second collagen layer was added to a well and allowed to polymerize. HUVECs were then fed with conditioned media. Five days later, the images were taken and analyzed using NIH ImageJ software. Number of sprouts per bead was determined as quantification of vessel formation.

\section{Statistics}

GraphPad Prism software (GraphPad Software, Inc. La Jolla, CA, USA) was used for statistical analyses. Unpaired two-tailed Student $t$ test was applied for comparison between two groups, and one-way ANOVA with the Tukey posttest was applied for comparison between three or more groups. Data were represented as mean $\pm \mathrm{SD}$ and were considered significant if $\mathrm{p}<0.05$.

\section{Results}

\section{Establishment of NSCLC model in mice}

Recent evidence encouraged us to investigate the role of TAM in NSCLC and the underlying molecular mechanisms. Thus, we first established a NSCLC model in mice that allows us to isolate and purify tumor cells from non-tumor cells in the tumor mass. We prepared an AAV carrying both luciferase and RFP reporter under a CMV promoter (Fig. 1A). This AAV was used to transduce an NSCLC cell line, A549. The transduced cells (transduction efficiency of $63.7 \pm 5.3 \%$ ) were purified by flow cytometry based on RFP expression (Fig. 1B). The purified transduced cells appeared to be red fluorescent in culture (Fig. 1C). The transduced/ labeled cells were i.v. injected to immune-deficient NOD/SCID mice to generate lung tumors. The successful model was confirmed by examination of presence of luciferase at lung area (Fig. 1D).

\section{NSCLC expresses high PLGF while TAM expresses high Flt-1 \\ VEGFR1/Flt-1 is the unique re-} ceptor for PLGF [6]. Moreover, previous studies have shown that Flt- 1 is expressed on endothelial cells and macrophages, we thus examined the expression pattern of PLGF and Flt1 on NSCLC cells and NSCLC-associated macrophages. We dissected the formed tumor in mice and dissociated it into single cell preparation for analysis by flow cytometry. We found that $65.5 \pm 6.3 \%$ of the cells in the tumor mass were red fluorescent (Fig. $2 \mathrm{~A}$ ), suggesting that these red cells were implanted A549 cells, while the non-red cells were non-tumor cells inside the tumor mass, e.g. endocrine cells, inflammatory cells and neurons. Next, we examined the PLGF levels in red vs non-red cells by RT-qPCR. Our data suggest that PLGF was predomi-

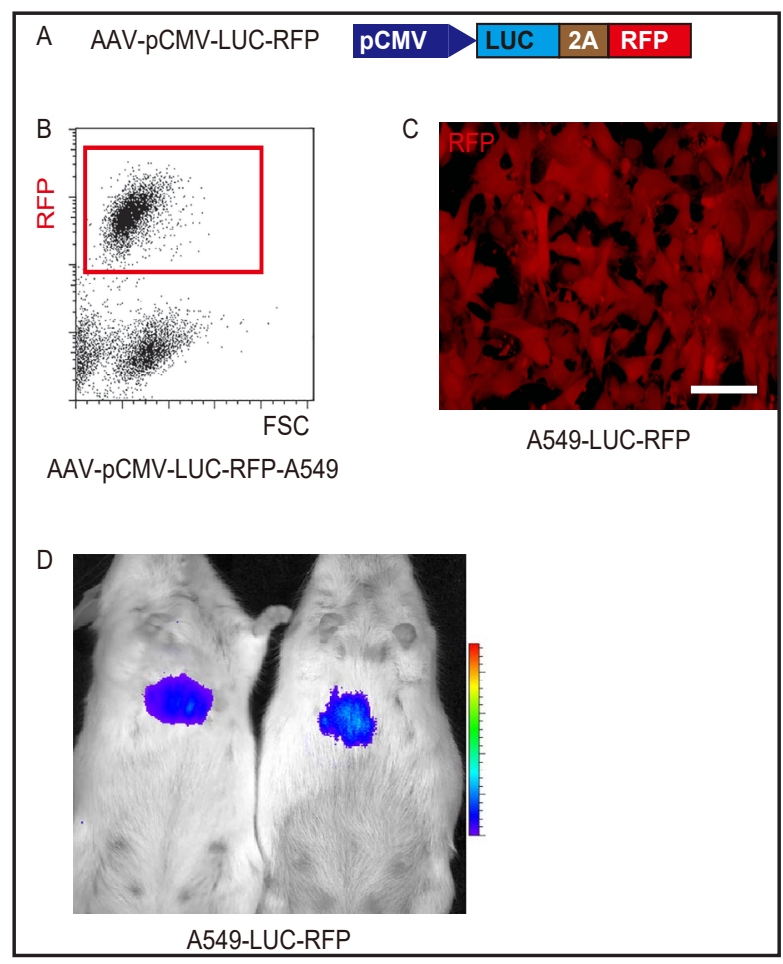

Fig. 1. Establishment of NSCLC in mice. (A) Schematic of an AAV vector, which carries both luciferase and RFP reporter under a CMV promoter. (B). The AAV-pCMV-LUCRFP was used to transduce an NSCLC cell line, A549. The transduced cells were purified by flow cytometry based on RFP expression. (C) The purified transduced cells appeared to be red fluorescent in culture. (D) The transduced/labeled AAV-pCMV-LUC-RFP-A549 cells were i.v. injected to immune-deficient NOD/SCID mice to generate lung cancer. The transplanted tumor cells were detected by bioluminescence. Scale bar is $10 \mu \mathrm{m}$. 
nantly expressed by tumor cells but not non-tumor cells (Fig. 2B). We then examined the Flt- 1 and red fluorescence in all the cells from the tumor mass. We found that seldom red cells expressed Flt-1, or the majority of the Flt-1+ cells were not red fluorescent (Fig. 2C). When we further examined these Flt-1 cells with CD31 (an endothelial cell marker) and F4/80 (a macrophage marker), we found that most of the Flt$1+$ cells were either CD31+ or F4/80+ and F4/80+ cells represented $\quad 72.4 \pm 5.6 \%$ of all Flt-1+ cells, while CD31+ cells represented $21.8 \pm 3.4 \%$ of all Flt $-1+$ cells (Fig. 2C-D). Furthermore, among all $\mathrm{F} 4 / 80+$ macrophages, $75.2 \pm 6.8 \%$ are CD163+, which represented a M2 or TAM phenotype (Fig. 2C, E).

NSCLC cells regulate macrophage polarization, which controls NSCLC cell growth

In order to understand the crosstalk between NSCLC cells and macrophages, we used a transwell co-culture system for A549

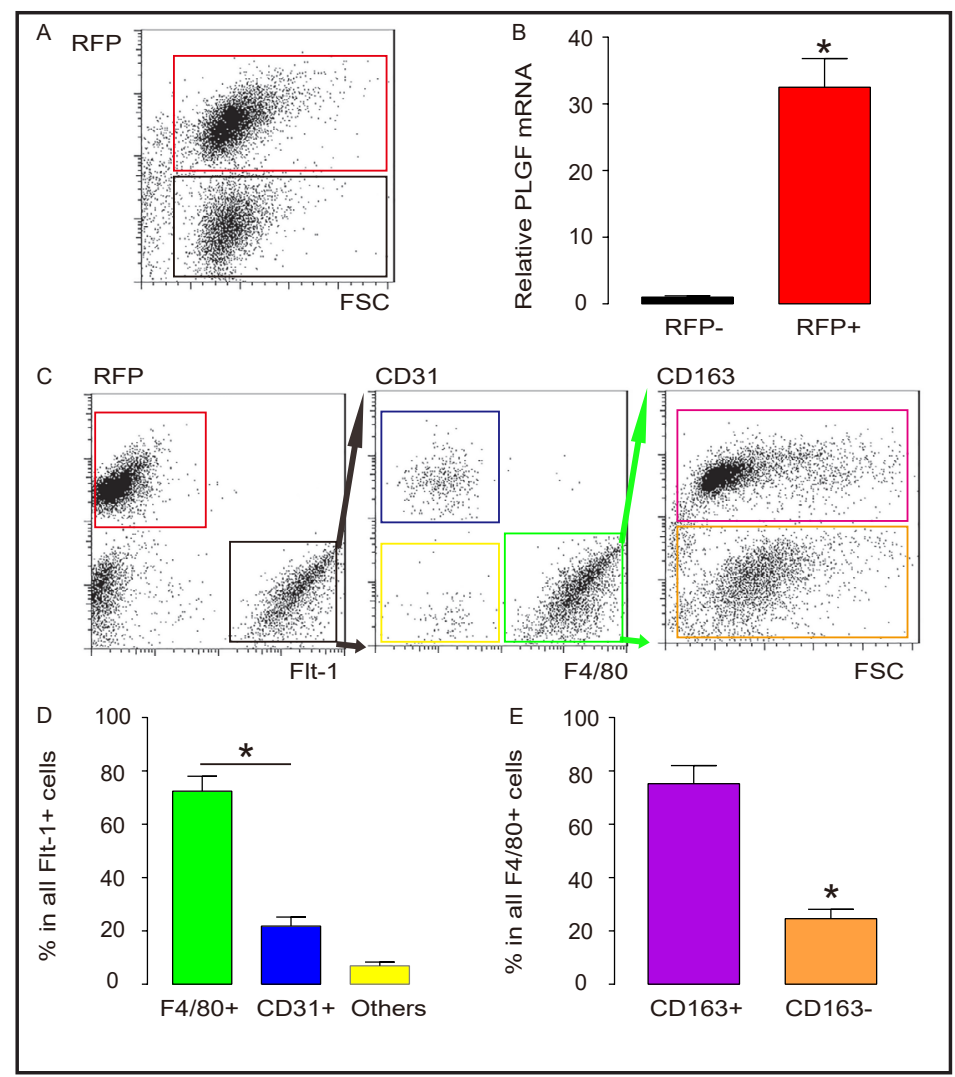

Fig. 2. NSCLC expresses high PLGF while TAM expresses high Flt-1. We dissected the formed tumor in mice and dissociated it into single cell preparation for analysis by flow cytometry. (A) Implanted tumor cells were detected by red fluorescence. (B) RT-qPCR for PLGF on red cells vs non-red cells. (C) Analyses of Flt-1 and red fluorescence on all the cells from the tumor mass. The Flt-1+ cells were analyzed for CD31 and F4/80 expression. The F4/80+ cells were analyzed for CD163 expression. All these flow cytometry analyses were shown by representative flow charts. (D) \% of F4/80+, CD31+, and other cells in all Flt-1+ cells, quantified by flow cytometry. (E) \% of CD163+, CD163- cells in all F4/80+ cells, quantified by flow cytometry. *: $p<0.05$. N=5. cells and bone-marrow-derived macrophages. To identify the role of PLGF in the setting, we used a condition in which PLGF was added to macrophages without presence of A549 cells. For these conditions, sFlt-1 was added as a loss-of-function control. Macrophages only, or A549 cells only condition was also applied as additional controls (Fig. 3A). Two days after culture, the growth of A549 cells by an MTT assay showed that macrophages significantly increased the A549 cell growth, in a PLGF/Flt-1 signaling-dependent manner, since sFlt- 1 significantly reduced the effects of macrophages on the augmentation of A549 cell growth (Fig. 3B). Similar results were obtained from manual counting of the culture cells (data not shown). Moreover, when we analyzed the subtype of macrophages by flow cytometry, we found that A549 cells significantly increased the M2/TAM macrophages, which was also significantly reduced by sFlt-1 (Fig. 3C-D). Furthermore, recombinant PLGF alone mimicked the effects of A549 cells on macrophage polarization (Fig. 3C-D). These data suggest that PLGF expression by A549 cells induces macrophage polarization, which is required for A549 cell growth. Hence, polarized macrophages may signal back to A549 cells to augment their growth. 
Fig. 3. NSCLC cells regulate macrophage polarization, which controls NSCLC cell growth. (A) Schematic of a transwell co-culture system for A549 cells and bone-marrow-derived macrophages (MФ). Group 1: MФ only. Group 2: A549 cells only. Group 3: $М \Phi$ and A549 cells. Group 4: MФ and A549 cells at presence of sFlt-1. Group 5: МФ only at presence of PLGF. Group 6: МФ only at presence of PLGF and sFlt-1. The incubation time was 2 days. A549 cells were then subjected to an MTT assay and M $\Phi$ were analyzed for CD163 by flow cytometry. (B) An MTT assay for growth of A549 cells during 2 days' culture. (C-D) Flow cytometry for CD163 on MФ, shown by quantification (C), and by representative flow charts (D). $*$ : $\mathrm{p}<0.05 . \mathrm{N}=5$.

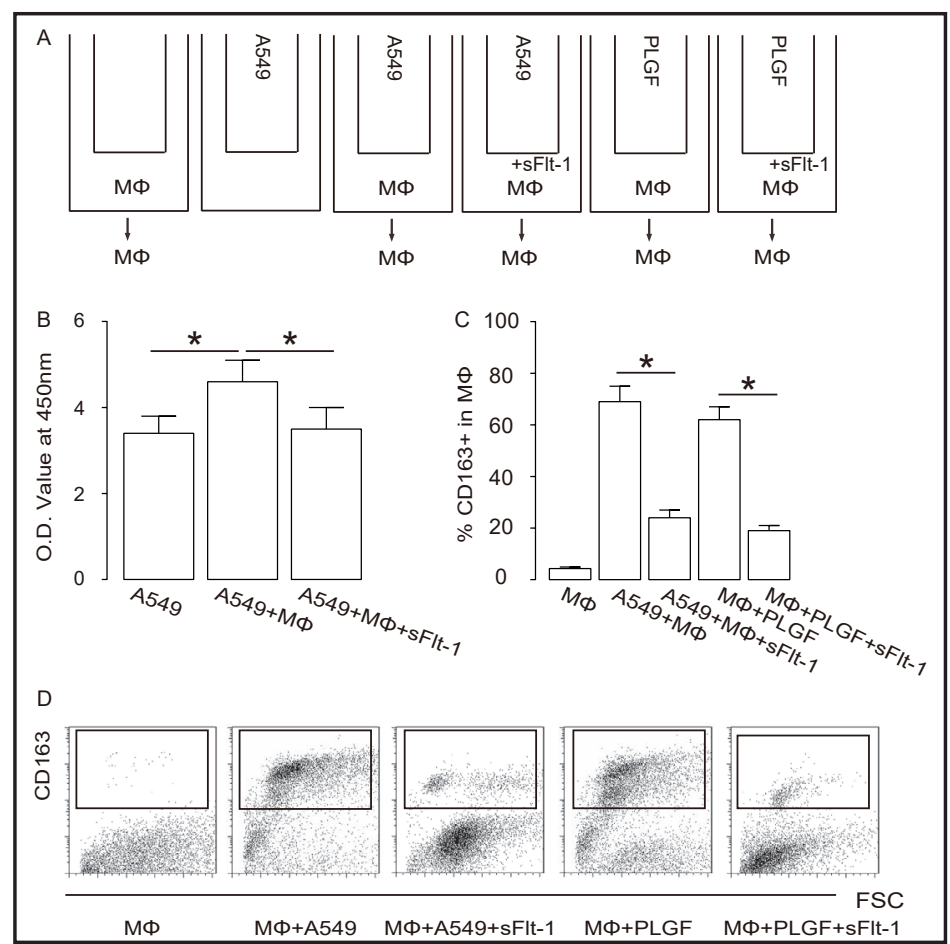

Polarized TAM promotes growth of NSCLC cells by TGF $\beta 1$

Next, we addressed the question on the signals of polarized TAM sent back to NSCLC cells. Since recent studies demonstrated a high level of TGF $\beta 1$ produced by M2/TAM cells, and TGF $\beta 1$ was a robust growth factor, we thus focused on TGF $\beta 1$. First, we confirmed that TGF $\beta 1$ was mainly produced by CD163+ M2/TAM, rather than by CD163- M1 macrophages (Fig. 4A). SB431542 (SB) is a specific inhibitor for TGF $\beta$ receptor I $[26,27]$, and was used as a loss-of-function control to be added into the co-culture of macrophages and A549 cells. Macrophages-only condition and macrophages with presence of SB were applied as additional controls (Fig. 4B). We found that when SB was added to macrophages without A549 cells, they had no effects on either macrophage polarization (Fig. 4C-D), M1 marker iNOS expression (Fig. 4E), or A549 cell growth in an MTT assay (Fig. 4F). Presence of SB in the interaction between macrophages and A549 cells did not alter macrophage polarization (Fig. 4C-D) or iNOS expression (Fig. 4E), suggesting that TGF $\beta$ receptor signaling did not play a role in the PLGF-induced macrophage polarization. However, the presence of SB significantly reduced the effects of macrophage polarization on A549 cell growth (Fig. 4F), suggesting that the polarized TAM may indeed promote growth of NSCLC cells by secretion of TGF 31 . In another word, polarized TAM may indeed promote growth of NSCLC cells in a TGF $\beta$ receptor signaling-dependent manner.

\section{PLGF-polarized TAM promotes neovascularization in vitro}

Finally, we examined if polarized macrophages may affect cancer-associated angiogenesis. Thus, we used a HUVEC collagen gel assay, in which HUVEC cells were cultured with the conditioned media (CM) from macrophages co-cultured with A549 cells with/ without presence of SB. Control media, recombinant TGF $\beta 1$ with/without SB were also included as conditions to compared (Fig. 5A). We found that the CM from macrophages cocultured with A549 cells significantly increased

Formation of tubular structures, which were completely blocked by presence of SB (Fig. $5 B-C)$. On the other hand, recombinant TGF $\beta 1$ in the culture media mimicked the effects of $\mathrm{CM}$ from macrophages co-cultured with $\mathrm{A} 549$ cells, which was similarly reduced by presence of SB (Fig. 5B-C). Together, these data suggest that PLGF-polarized TAM may promote neovascularization in a TGF $\beta$ receptor signaling-dependent manner. 
Fig. 4. Polarized TAM promotes growth of NSCLC cells by TGFß1. (A) RT-qPCR for TGF $\beta 1$ on CD163+ vs CD163- macrophages. (B) Schematic of a transwell co-culture system for A549 cells and bonemarrow-derived macrophages (МФ). Group 1: МФ only. Group 2: MФ treated with SB. No A549 cells. Group 3: МФ and A549 cells. Group 4: MФ and A549 cells at presence of SB431542 (SB). The incubation time was 2 days. A549 cells were then subjected to an MTT assay and $\mathrm{M} \Phi$ were analyzed for CD163 by flow cytometry. (C-D) Flow cytometry for CD163 on MФ, shown by representative flow charts (C), and by quantification (D). (E) RTqPCR for iNOS in MФ. (F) An MTT assay for growth of A549 cells during 2 days' culture.*: $\mathrm{p}<0.05$. NS: non-significant. $\mathrm{N}=5$.
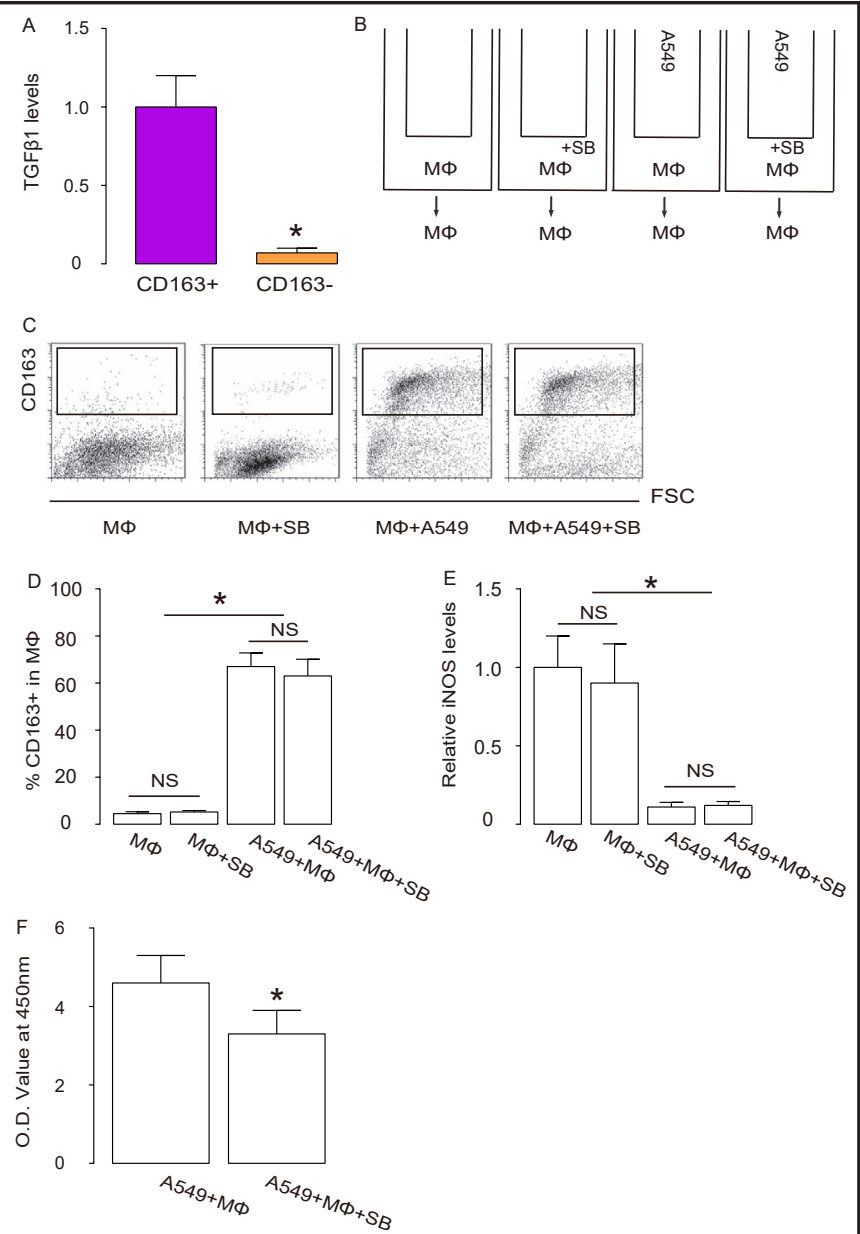

Fig. 5. PLGF-polarized TAM promotes neovascularization in vitro. (A) Schematic of the experiment. Group 1: control media (CTL). Group 2: conditioned media (CM) from a transwell co-culture system for A549 cells and bone-marrow-derived macrophages (MФ). Group 3: CM from a transwell coculture system for A549 cells and M $\Phi$ at presence of SB. Group 4: control media plus TGF $\beta 1$. Group 5: control media plus TGF $\beta 1$ and SB431542 (SB). The incubation time for co-culture system was 2 days. The media were applied to culture HUVEC in a collagen gel assay. (B-C). HUVEC tubular structure formation by M2-polarized macrophages was evaluated and shown by representative images (B), and by quantification (C). *: $\mathrm{p}<0.05$. $\mathrm{N}=5$.

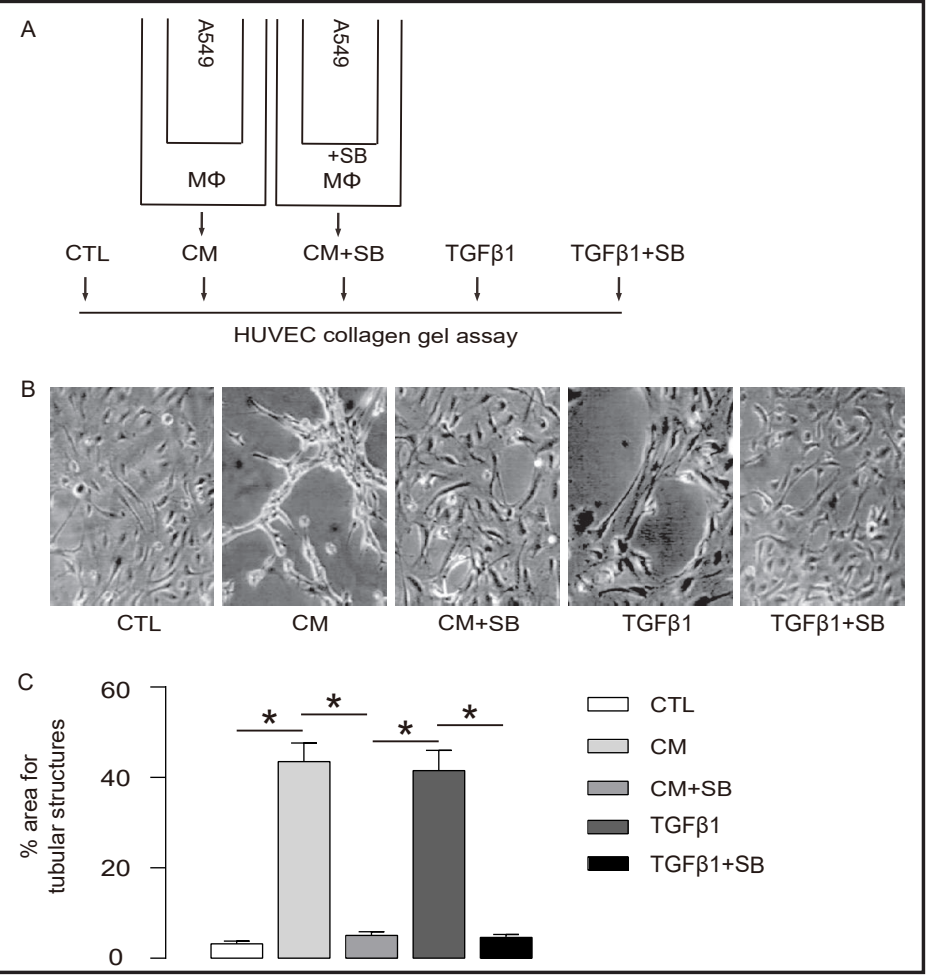




\section{Discussion}

Elucidation of the molecular mechanisms underlying the growth and invasion of NSCLC may essentially improve its prognosis and generate novel therapies. Previous studies have highlighted the importance of cancer-associated angiogenesis to the growth and metastasis of the primary tumor, in which tumor cells may produce and secrete angiogenetic molecules like VEGF and PLGF to increase tumor capillary permeability and promote endothelial cell proliferation and survival inside the tumor mass [28]. However, the regulation of angiogenesis involves a complex network comprised of tumor cells, inflammatory cells, mesenchymal cells, endothelial cells and other cell types. The detailed molecular signaling remains unclear [29, 30]. However, TAM has been recently recognized as a key player in the signaling transduction that regulates tumor angiogenesis [17-20,29-31], although the molecular activation of TAM in NSCLC remains elusive.

Here, we addressed the paracrine manner of the PLGF from NSCLC cells. Our data showed that the major sources for the unique PLGF receptor -Flt-1- were endothelial cells and macrophages inside the NSCLC tumor mass. While PLGF/Flt-1 signaling has a demonstrate role in the augmentation of tumor vessel growth, we hypothesized that this signaling may also affect tumor-associated angiogenesis via macrophages, since macrophages have a higher number than endothelial cells inside the tumor mass [7-10] and TAM are known to be promoting vascularization [17-20, 29-31].

In order to confirm that NSCLC cells trigger macrophage polarization via PLGF itself, we used PLGF alone as a gain-of-function control. Moreover, we added sFlt-1 into the co-culture system as a loss-of-function control. Our data on growth of NSCLC cells and macrophage polarization suggest that PLGF/Flt-1 signaling regulates macrophage polarization, which subsequently controls the growth of NSCLC cells. In the next co-culture system, we added $\mathrm{SB}$ into the co-culture system to inhibit TGF $\beta$ receptor signaling as a loss-of-function control. Since the macrophage polarization was unaltered, but the growth of NSCLC cells were inhibited, these data suggest that TGF $\beta$ receptor signaling mediated the effects of polarized macrophages on NSCLC cells, rather than the control of macrophage polarization by NSCLC cells. Finally, application of SB significantly reduced the effects of macrophages or TGF $\beta 1$ on vessel sprouting in the collagen gel assay, suggesting that TGF $\beta$ receptor signaling not only mediates the effects of TAM on NSCLC cell growth, but also the effects of TAM on the angiogenesis. Since the amino acids of Flt-1 are very conserved in mouse and human [32], the slightly diverge amino acid sequence appeared to have minimal effects on the interaction between mouse bone marrow-derived macrophages and human lung cancer cells through PLGF/Flt-1 regulatory axis.

A recent study showed that M2 macrophages were recruited to the injured pancreas, and produced and secreted high levels of TGF $\beta 1$ to promote beta cell proliferation [22]. In another report, mesenchymal stem cells transplanted to injured heart not only recruit macrophages to enhance angiogenesis to promote regeneration of cardiac muscle, but also secrete BMP7 to contradict the fibrogenic effect of TGF $\beta 1$ by macrophages [23]. Both studies highlighted that M2 macrophages secreted high TGF $\beta 1$, which was confirmed in the current study. Hence, the M2 macrophages have a trophic effect on cell growth, which may be beneficial or detrimental for tissue regeneration during injury, but appears to be detrimental in tumor.

Zhou et al. recently showed that larynx carcinoma regulates TAM through PLGF signaling [24], in which the authors found that PLGF secreted by larynx carcinoma cells triggered macrophage polarization to TAM to produce MMP9. However, a subsequent effect of macrophage polarization on the growth of cancer cells and angiogenesis was not examined in this study [24]. Here, we addressed these questions and presented a model to explain the crosstalk among NSCLC cells, endothelial cells and TAM that regulates tumorigenesis of NSCLC.

To the best of our knowledge, it is so far the first study to show PLGF/Flt-1 signaling and TGF $\beta$ receptor signaling mediate the two phases of the regulation processes respectively. 
Further delineation of the detailed molecular mechanisms that control these processes may substantially improves our understanding of the carcinogenesis of NSCLC.

\section{Acknowledgments}

This work is supported by Hei Long Jiang Postdoctoral Foundation(LBH-Q16179), Hei Long Jiang Health and Family Planning Commission(2016-113), Nn10 Program of Harbin Medical University Cancer Hospital, Wu Jieping Fund, and Qilu Cancer Research Fund.

\section{Disclosure Statement}

The authors have declared that no competing interests exist.

\section{References}

1 Carter CA, Zeman K, Day RM, Richard P, Oronsky A, Oronsky N, Lybeck M, Scicinski J, Oronsky B: Addressing the elephant in the room, therapeutic resistance in non-small cell lung cancer, with epigenetic therapies. Oncotarget 2016;7:40781-40791.

-2 Taverna S, Giallombardo M, Gil-Bazo I, Carreca AP, Castiglia M, Chacartegui J, Araujo A, Alessandro R, Pauwels P, Peeters M, Rolfo C: Exosomes isolation and characterization in serum is feasible in non-small cell lung cancer patients: critical analysis of evidence and potential role in clinical practice. Oncotarget 2016;7:28748-28760.

-3 Hall RD, Le TM, Haggstrom DE, Gentzler RD: Angiogenesis inhibition as a therapeutic strategy in non-small cell lung cancer (NSCLC). Transl Lung Cancer Res 2015;4:515-523.

4 Dufour A, Overall CM: Missing the target: matrix metalloproteinase antitargets in inflammation and cancer. Trends Pharmacol Sci 2013;34:233-242.

-5 Pottgen C, Eberhardt W, Stamatis G, Stuschke M: Definitive radiochemotherapy versus surgery within multimodality treatment in stage III non-small cell lung cancer (NSCLC) - a cumulative meta-analysis of the randomized evidence. Oncotarget 2017;8:41670-41678.

6 Ferrara N: Vascular endothelial growth factor. Arterioscler Thromb Vasc Biol 2009;29:789-791.

7 Fischer C, Jonckx B, Mazzone M, Zacchigna S, Loges S, Pattarini L, Chorianopoulos E, Liesenborghs L, Koch M, De Mol M, Autiero M, Wyns S, Plaisance S, Moons L, van Rooijen N, Giacca M, Stassen JM, Dewerchin M, Collen D, Carmeliet P: Anti-PlGF inhibits growth of VEGF(R)-inhibitor-resistant tumors without affecting healthy vessels. Cell 2007;131:463-475.

-8 Autiero M, Waltenberger J, Communi D, Kranz A, Moons L, Lambrechts D, Kroll J, Plaisance S, De Mol M, Bono F, Kliche S, Fellbrich G, Ballmer-Hofer K, Maglione D, Mayr-Beyrle U, Dewerchin M, Dombrowski S, Stanimirovic D, Van Hummelen P, Dehio C, Hicklin DJ, Persico G, Herbert JM, Shibuya M, Collen D, Conway EM, Carmeliet P: Role of PIGF in the intra- and intermolecular cross talk between the VEGF receptors Flt1 and Flk1. Nat Med 2003;9:936-943.

-9 Huo X, Li Y, Jiang Y, Sun X, Gu L, Guo W, Sun D: Inhibition of Ocular Neovascularization by Co-Inhibition of VEGF-A and PLGF. Cell Physiol Biochem 2015;35:1787-1796.

10 Jiang H, Wu X, Wang H, Huang C, Zhang L: Combined Anti-PLGF and Anti-Endostatin Treatments Inhibit Ocular Hemangiomas. Cell Physiol Biochem 2015;36:930-936.

$\checkmark 11$ Tudisco L, Orlandi A, Tarallo V, De Falco S: Hypoxia activates placental growth factor expression in lymphatic endothelial cells. Oncotarget 2017;8:32873-32883.

12 Li J, Ying H, Cai G, Guo Q Chen L: Pre-Eclampsia-Associated Reduction in Placental Growth Factor Impaired Beta Cell Proliferation Through PI3k Signalling. Cell Physiol Biochem 2015;36:34-43.

13 Song N, Liu H, Ma X, Zhang S: Placental Growth Factor Promotes Metastases of Ovarian Cancer Through MiR-543-Regulated MMP7. Cell Physiol Biochem 2015;37:1104-1112.

14 Song N, Liu H, Ma X, Zhang S: Placental Growth Factor Promotes Ovarian Cancer Cell Invasion via ZEB2. Cell Physiol Biochem 2016;38:351-358. 


\section{Cellular Physiology Cell Physiol Biochem 2018;47:2534-2543 \begin{tabular}{l|l} 
and Biochemistry Published online: July 10, 2018 & $\begin{array}{l}\text { C) } 2018 \text { The Author(s). Published by S. Karger AG, Basel } \\
\text { www.karger.com/cpb }\end{array}$ \\
\hline
\end{tabular}}

He et al.: PLGF Regulates Crosstalk Between NSCLC and TAM

15 Zhang J, Chen A, Wu Y, Zhao Q: Placental Growth Factor Promotes Cardiac Muscle Repair via Enhanced Neovascularization. Cell Physiol Biochem 2015;36:947-955.

16 Zhang W, Zhang T, Lou Y, Yan B, Cui S, Jiang L, Han B: Placental Growth Factor Promotes Metastases of NonSmall Cell Lung Cancer Through MMP9 . Cell Physiol Biochem 2015;37:1210-1218.

17 Flavell RA, Sanjabi S, Wrzesinski SH, Licona-Limon P: The polarization of immune cells in the tumour environment by TGFbeta. Nat Rev Immunol 2010;10:554-567.

18 Gordon S, Taylor PR: Monocyte and macrophage heterogeneity. Nat Rev Immunol 2005;5:953-964.

19 Pollard JW: Trophic macrophages in development and disease. Nat Rev Immunol 2009;9:259-270.

20 Grunewald M, Avraham I, Dor Y, Bachar-Lustig E, Itin A, Jung S, Chimenti S, Landsman L, Abramovitch R, Keshet E: VEGF-induced adult neovascularization: recruitment, retention, and role of accessory cells. Cell 2006;124:175-189.

21 Lin L, Chen YS, Yao YD, Chen JQ, Chen JN, Huang SY, Zeng YJ, Yao HR, Zeng SH, Fu YS, Song EW: CCL18 from tumor-associated macrophages promotes angiogenesis in breast cancer. Oncotarget 2015;6:34758-34773.

22 Xiao X, Gaffar I, Guo P, Wiersch J, Fischbach S, Peirish L, Song Z, El-Gohary Y, Prasadan K, Shiota C, Gittes GK: M2 macrophages promote beta-cell proliferation by up-regulation of SMAD7. Proc Natl Acad Sci U S A 2014;111:E1211-1220.

23 Wang M, Zhang G, Wang Y, Liu T, Zhang Y, An Y, Li Y: Crosstalk of mesenchymal stem cells and macrophages promotes cardiac muscle repair. Int J Biochem Cell Biol 2015;58:53-61.

-24 Zhou X, Qi Y: Larynx carcinoma regulates tumor-associated macrophages through PLGF signaling. Scientific reports 2015;5:10071.

25 Giard DJ, Aaronson SA, Todaro GJ, Arnstein P, Kersey JH, Dosik H, Parks WP: In vitro cultivation of human tumors: establishment of cell lines derived from a series of solid tumors. J Natl Cancer Inst 1973;51:14171423.

-26 Matsuyama S, Iwadate M, Kondo M, Saitoh M, Hanyu A, Shimizu K, Aburatani H, Mishima HK, Imamura T, Miyazono K, Miyazawa K: SB-431542 and Gleevec inhibit transforming growth factor-beta-induced proliferation of human osteosarcoma cells. Cancer Res 2003;63:7791-7798.

-27 Inman GJ, Nicolas FJ, Callahan JF, Harling JD, Gaster LM, Reith AD, Laping NJ, Hill CS: SB-431542 is a potent and specific inhibitor of transforming growth factor-beta superfamily type I activin receptor-like kinase (ALK) receptors ALK4, ALK5, and ALK7. Mol Pharmacol 2002;62:65-74.

28 Albini A, Tosetti F, Li VW, Noonan DM, Li WW: Cancer prevention by targeting angiogenesis. Nat Rev Clin Oncol 2012;9:498-509.

29 Welti J, Loges S, Dimmeler S, Carmeliet P: Recent molecular discoveries in angiogenesis and antiangiogenic therapies in cancer. J Clin Invest 2013;123:3190-3200.

-30 Yang Y, Sun M, Wang L, Jiao B: HIFs, angiogenesis, and cancer. J Cell Biochem 2013;114:967-974.

-31 Mantovani A, Sica A: Macrophages, innate immunity and cancer: balance, tolerance, and diversity. Curr Opin Immunol 2010;22:231-237.

32 Meyer RD, Mohammadi M, Rahimi N: A single amino acid substitution in the activation loop defines the decoy characteristic of VEGFR-1/FLT-1. J Biol Chem 2006;281:867-875. 